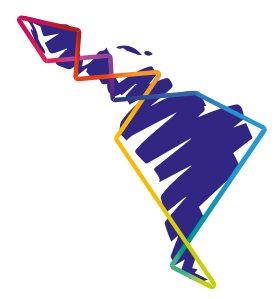

\title{
Violencia institucional y violación del derecho a la salud: Elementos para repensar la exigibilidad del derecho a la salud en el México actual
}

\author{
Institutional violence and violation \\ of the right to health: Elements \\ to rethink the demandability of \\ the right to health in present-day \\ Mexico
}

Clara Bellamy'

\begin{abstract}
Resumen
El objetivo de la presente investigación fue generar evidencias sobre elementos que puedan constituir obstáculos en el ejercicio del derecho a la salud y, a la vez, de documentar las violaciones de dicho derecho y caracterizar las principales formas de violencia institucional que sufren el público usuario de los servicios públicos de salud. Se analizaron las recomendaciones de la Comisión Nacional de Derechos Humanos (CNDH) publicadas en su gaceta mensual entre los años 2000 y 2016, solo en los casos referentes a la salud. Se realizó un análisis cuantitativo de frecuencias simples y relaciones bivariadas, con lo cual se conformaron cuatro perfiles poblacionales: mujeres embarazadas, mujeres no embarazadas, hombres y casos colectivos. Posteriormente, se hizo un análisis de contenido de los relatos jurídicos para cada grupo y se analizaron por medio de cuatro categorías analíticas: la espera, la cadena de errores, la violencia obstétrica y la exclusión. Caracterizamos los patrones en la violación del derecho a la salud y debatimos contra los argumentos que sustentan que la mayoría de los errores se deben principalmente a problemas de infraestructura. A pesar de considerar que las carencias, en efecto, influyen en la atención, estas no son las que más afectan: Un hallazgo del estudio es que, en un gran número de casos de las quejas presentadas ante la $\mathrm{CNDH}$, se refieren a elementos relacionados con el clasismo, el racismo y el sexismo, perpetrados a través prácticas de discriminación, de autoritarismo y de exclusión social.
\end{abstract}

1 Dra. en Ciencias de la Salud, UNAM, Programa de Becas Posdoctorales en la UNAM, Becaria del CRIM asesorada por el Dr. Roberto Castro. Institución: CRIM-UNAM. 
Palabras clave: derecho a la salud, derechos humanos, violencia institucional, violencia obstétrica, exclusión.

\section{Summary}

The objective of the present investigation was to generate evidence on elements that may constitute obstacles in the exercise of the right to health, while documenting violations of said right and characterizing the main forms of institutional violence suffered by users of public health services. The recommendations of the Comision Nacional de Derechos Humanos (CNDH) (National Human Rights Commission), published in its monthly gazette between 2000 and 2016, were analyzed for cases pertaining to health. A quantitative analysis of simple frequencies and bivariate relationships was carried out and four population profiles were established: pregnant women, non-pregnant women, men and collective cases. Subsequently, a content analysis of the legal narrations for each group was performed and analyzed through four analytical categories: (attention) waiting time, chain of errors, obstetric violence and exclusion. We characterize the patterns present in the violation of the right to health and debate against the arguments that support the notion that most of the errors are mainly due to infrastructure problems. In spite of considering that infrastructure deficiencies effectively influence user attention, a finding of the investigation is that, in a great number of complaint cases presented before the $\mathrm{CNDH}$, infrastructure deficiencies are not the main element, but instead, are superseded by elements related to classism, racism and sexism, perpetrated through practices of discrimination, authoritarianism and social exclusion.

Keywords: Right to health; Human rights; Institutional violence; Obstetric violence; Exclusion.

\section{Introducción}

El derecho a la salud se contempla en distintos instrumentos jurídicos; está establecido en el artículo $4^{\circ}$ constitucional, así como en diversas leyes, pactos, declaraciones y convenciones internacionales. Sin embargo, existe una agenda pendiente, ya que muchos sectores de la población mundial no cuentan aún con las condiciones para ejercer plenamente sus derechos, a pesar de ser el objeto de los discursos sobre estos (De Sousa Santos, 2014).

En México existen diversos organismos encargados de proteger y defender los derechos humanos ante violaciones por parte de autoridades administrativas o personal de los servicios públicos. Además de los tribunales, que atienden denuncias y demandas, la Comisión Nacional de Derechos Humanos (CNDH) y las Comisiones Estatales de Derechos Humanos $(\mathrm{CEDH})$ son una fuente privilegiada para observar el fenómeno de la violación de los derechos humanos.

Existen diversas situaciones de violencia contra el público usuario de los servicios de salud que están estrechamente vinculadas con la violación del derecho a la 
salud, donde la propia noción de derecho está ausente de su ejercicio, tanto entre personas usuarias como entre profesionales de la salud (Rueda, 2013).

Las violaciones del derecho a la salud se vinculan con la violencia institucional definida como las "prácticas estructuradas de violación de derechos por parte de funcionarios pertenecientes a fuerzas de seguridad, fuerzas armadas, servicios penitenciarios y efectores de salud en contextos de restricción de autonomía y/o [sic] libertad" (Armida, Cassino, Ciarniello, \& Witis, s. f., p. 6). Son manifestaciones de violencia en las que el Estado, por acción u omisión, tiene responsabilidad directa; pero también incluye acciones de discriminación o de obstáculo en el ejercicio y goce de los derechos (Bodelón, 2014; Perelman \& Trufó, 2017).

La magnitud de las violaciones del derecho a la salud es aún desconocida. De todas las quejas que recibe y resuelve la $\mathrm{CNDH}$, las relativas al área de la salud se encuentran entre las más numerosas, incluso por encima de las quejas relacionadas con abusos por parte del ejército, la marina, la policía y las instituciones de procuración de justicia.

Si bien sigue pendiente una medición objetiva de la magnitud de las violaciones al derecho a la salud, sí es posible profundizar cualitativamente en la descripción de este problema. En este trabajo presentamos un análisis de las recomendaciones emitidas por la CNDH entre 2000 y 2016 en el área de la salud, con el fin de ofrecer una clasificación y una caracterización de los principales patrones de violación de derechos que se observan en ese campo. Mostraremos que las violaciones a este derecho son básicamente de tipo social y se deben, no tanto a problemas de infraestructura, como a problemas de clasismo, de discriminación, de autoritarismo, violación de la autonomía en las decisiones, violencia de género y de exclusión social.

\section{Metodología}

Se realizó una sistematización de las recomendaciones en materia de salud emitidas por la CNDH, publicadas mensualmente en su gaceta (órgano oficial de difusión de esta institución), durante el periodo 2000 a 2016. Tales recomendaciones presentan un formato jurídico que incluye el relato de los hechos, formulado desde la perspectiva de la parte que presenta la queja. En términos generales, la estructura de tales documentos incluye los siguientes rubros: síntesis, hechos, evidencias, observaciones, situación jurídica y, finalmente, las recomendaciones que emitió la CNDH para cada caso. Sin embargo, el formato de presentación de estas recomendaciones ha ido cambiando a lo largo del tiempo, lo que explica que no tengamos el mismo número de casos para cada una de las variables. 
Se revisaron todos los casos en cada una de las gacetas para identificar los relacionados propiamente con el tema de investigación. Las gacetas revisadas corresponden a los números 114 a la 317, en los cuales se identificó un total de 228 recomendaciones de la CNDH en materia de salud ${ }^{2}$. Cabe destacar que las recomendaciones que se emiten corresponden a quejas en las cuales la CNDH estimó que hubo una violación de derechos humanos (en nuestro caso, del derecho a la salud), si bien el número total de quejas que se reciben en esa institución es mucho mayor.

Se capturaron datos de variables sociodemográficas como sexo, edad, Estado, municipio, sector de salud, etc. Otras relativas a la búsqueda de atención, el diagnóstico, el desenlace del caso, el número de búsquedas, el número de médicos involucrados, las especialidades de estos y otro personal médico, entre otras. Las recomendaciones se desagregaron por categoría, por el periodo del ombudsman y por el número total de recomendaciones emitidas. También se utilizaron los indicadores desagregados de negligencia, imprudencia e impericia ${ }^{3}$. Y se profundizó en el análisis de la violencia obstétrica, dada la alta frecuencia de estos casos como la propia CNDH la ha identificado , y por lo cual emitió en 2017 la recomendación general 31 "Sobre la violencia obstétrica en el Sistema Nacional de Salud".

A partir de la base de datos original, se recategorizaron algunas variables, se crearon nuevas y se analizaron los datos a través de medidas de tendencia central y de dispersión, así como el cruce de variables en tablas de contingencia o de diferencias de medias. La base se conformó con un total de 124 variables.

Para el análisis de las recomendaciones, primero se hizo un análisis exploratorio de las frecuencias simples de todas las variables de la base de datos. En principio, al utilizar la variable sexo se pretendía dividir el análisis en hombres y mujeres. Pero, al identificar que la mayoría eran mujeres y de estas, la mayor parte correspondían a mujeres en la etapa del embarazo, parto o puerperio, se decidió segmentar a las mujeres en embarazadas y no embarazadas, luego a los hombres y, finalmente, se decidió separar los casos colectivos por no pertenecer a ninguna de las tres opciones anteriores. De tal manera conformamos los cuatro perfiles poblacionales, con los cuales se realizó de nuevo el análisis de frecuencias simples y se identificaron diferencias a partir de la lectura del relato jurídico. El análisis estadístico nos sirvió para reconocer algunos indicadores y frecuencias que dieron pie al análisis de contenido de los relatos.

2 Se excluyeron del análisis las impugnaciones.

3 La negligencia es la omisión o demora injustificada en la actuación del médico o la actuación perezosa, con carencia o de constancia profesional; la impericia es la ignorancia inexcusable, y la imprudencia es la actuación temeraria o precipitada (Comisión Nacional de los Derechos Humanos, 2017). 


\section{Resultados}

\section{Caracterización general de la muestra en perfiles poblacionales}

Como ya señalamos, en el período 2000 al 2016 encontramos en total 228 recomendaciones de la CNDH referidas a violaciones al derecho a la salud, los cuales clasificamos en cuatro perfiles poblacionales: 1 ) hombres, 2) mujeres embarazadas, 3) mujeres no embarazadas y 4) casos colectivos.

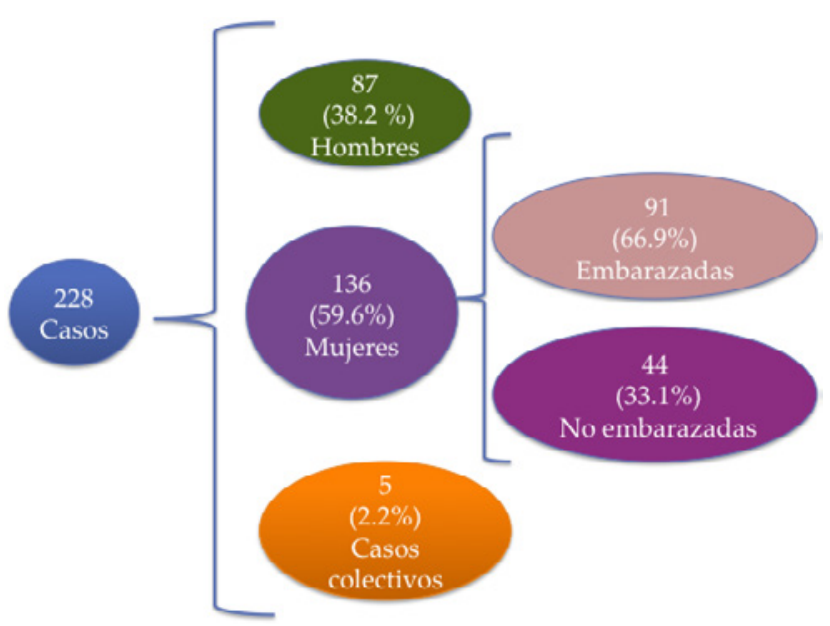

Figura 1. Distribución de los perfiles poblacionales de los casos de las recomendaciones en salud de la gaceta de la CNDH 2000-2016. Elaboración propia con datos de las recomendaciones en salud de la gaceta de la CNDH 2000-2016.

En la distribución poblacional respecto a las quejas de violación del derecho a la salud encontramos un marcado componente de género, dado que el $59.6 \%$ de las recomendaciones analizadas corresponden a quejas presentadas por mujeres $\mathrm{y}$, de ellas, el $66.9 \%$ es específicamente en la etapa del embarazo. 


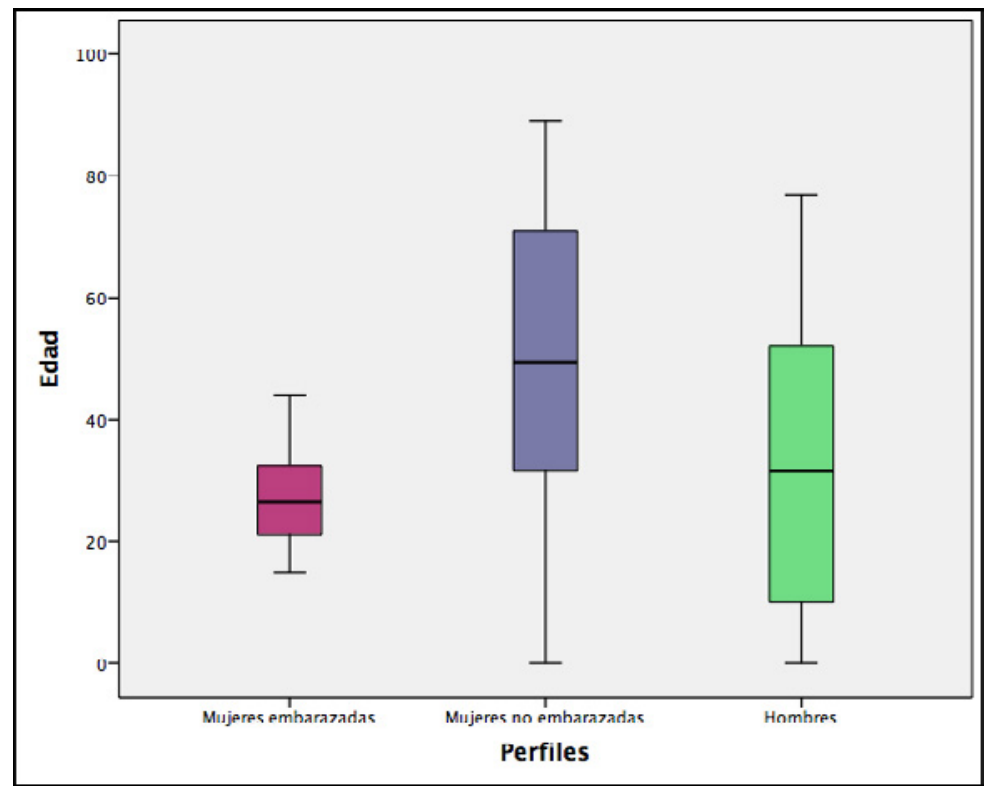

Figura 2. Distribución de los perfiles poblacionales de los casos de las recomendaciones en salud de la gaceta de la CNDH 2000-2016 según edad.

Fuente: Elaboración propia con datos de las recomendaciones de la CNDH en la gaceta 2000 $-2016$.

Las mujeres embarazadas se ubicaron en el rango de edad reproductiva (15-49 años) con una media de 27.1 años. Las mujeres no embarazadas presentaron una media de 49.3 años. En el grupo de hombres la edad promedio fue de 31.3 años. Como se aprecia en la figura 2, la dispersión en las mujeres no embarazadas y en los hombres es mayor que en el grupo de las embarazadas, la variabilidad de edad en ambos grupos oscila entre un mes de edad a los 89 años, aunque en las mujeres no embarazadas los casos se concentran más en edades avanzadas y, en los hombres, al contrario. 

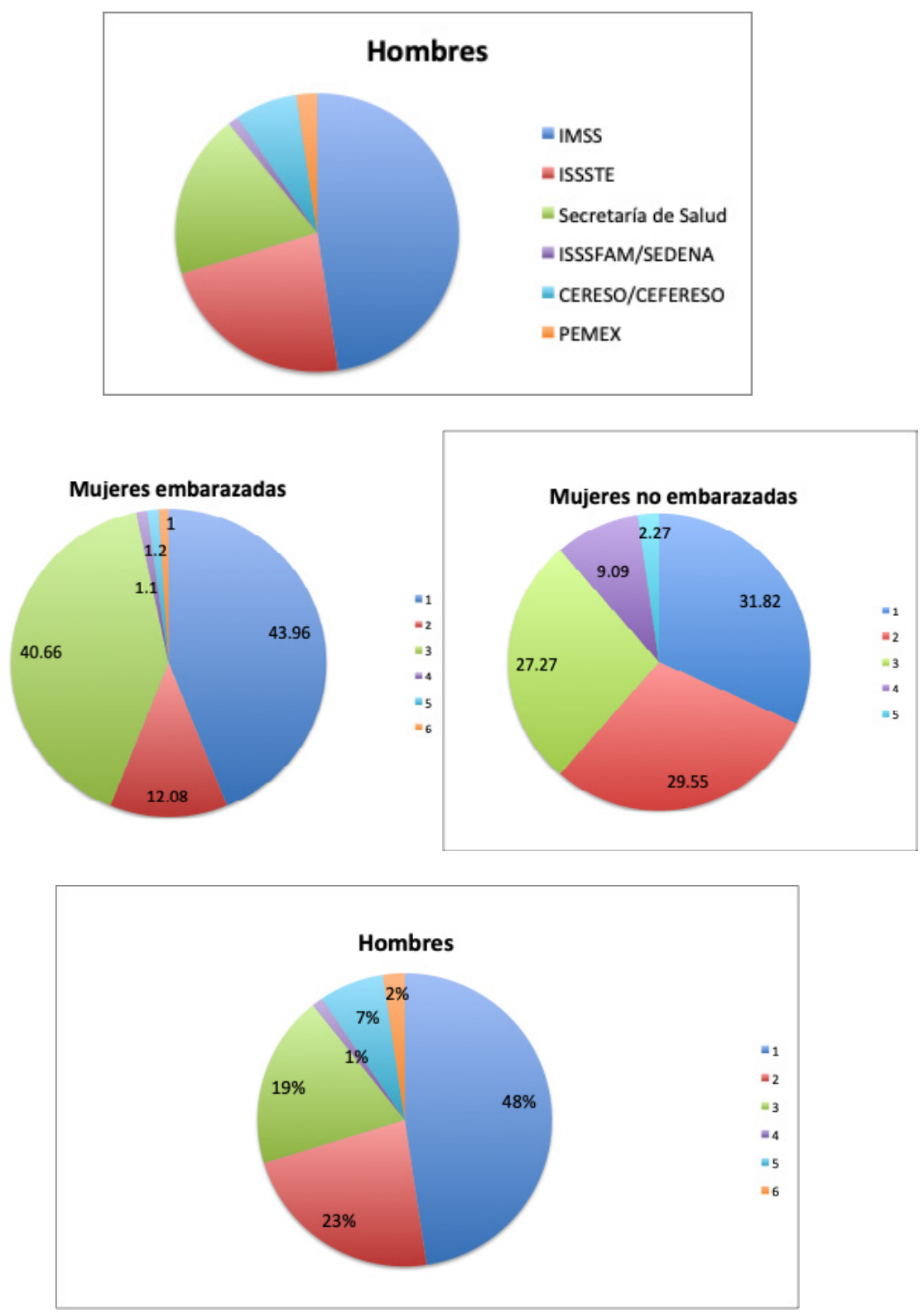

Figura 3. Distribución de los perfiles poblacionales de los casos de las recomendaciones en salud de la gaceta de la CNDH 2000-2016 según sector de salud.

Fuente: Elaboración propia con datos de las recomendaciones de la CNDH en la gaceta 2000 $-2016$. 
Respecto a la utilización de los servicios de salud públicos, encontramos que en las mujeres embarazadas el porcentaje más alto fue del Instituto Mexicano del Seguro Social (IMSS) en un 43.9\%, seguido de la Secretaría de Salud (SSA) en un $40.6 \%$, y $12 \%$ en el Instituto de Servicios Sociales y Salud de los Trabajadores al Servicio del Estado (ISSSTE). ${ }^{4}$ En las mujeres no embarazadas los porcentajes entre el IMSS, la SSA y el ISSSTE fueron similares con $31.8 \%, 27.2 \%$ y $29.5 \%$, respectivamente. Encontramos, además, un $9 \%$ en el Instituto de Seguridad Social para las Fuerzas Armadas (ISSSFAM), porcentaje que resulta más alto que el de los hombres. En los hombres el porcentaje más alto fue del IMSS con un $47.6 \%$, seguido del ISSSTE en un $22.6 \%$ y de la SSA en un $19 \%$, además hay un $7 \%$ en los Centros de Rehabilitación Social (CERESOS) y Centros Federales de Rehabilitación Social (CEFERESOS).

\section{Caracterización del perfil de violación del derecho a la salud}

$\mathrm{Al}$ analizar las recomendaciones encontramos violaciones del derecho a la salud que caracterizamos en torno a cuatro categorías analíticas: la espera, la cadena de errores, la violencia obstétrica y la exclusión. Las cuatro categorías se concatenan de distintas maneras en cada uno de los casos, pero encontramos perfiles generales que comparten dichas quejas.

\section{La espera}

Retomamos la propuesta de Auyero (2013) quien indaga cómo la espera se constituye en una herramienta de control y dominación del Estado sobre los sectores pobres. Mediante este mecanismo, los grupos marginados generan una subjetividad respecto a la espera, mediante la cual aprenden a ser sumisos y a esperar, a lidiar con la incertidumbre y con las arbitrariedades de la burocracia que con frecuencia impone trámites cambiantes que funcionan como obstáculos para el ejercicio del derecho a la salud. El análisis de la espera se constituye a partir de la peregrinación por los servicios; la negación de la atención; el peligro en la demora o el abandono del paciente; la generación de confusiones, errores, demoras interminables e indiferencia burocrática, con el consiguiente peloteo al que quedan sujetas las personas, en el cual podemos advertir rituales públicos de degradación (Auyero, 2013).

4 El Instituto Mexicano del Seguro Social (IMSS) atiende a trabajadores del sector privado; la Secretaría de Salud (SSA), a personas que no cuentan con ningún servicio de seguridad social; y el Instituto de Servicios Sociales y Salud de los Trabajadores al Servicio del Estado (ISSSTE), a trabajadores del sector público. 
También analizamos los relatos en torno a la responsabilidad institucional, la que Auyero analiza como velo místico, que remite a una analogía del fetichismo de la mercancía, en la cual se oculta la responsabilidad de las acciones humanas por medio del funcionamiento del sistema y reduce las relaciones entre objetos y no entre personas. De tal forma se desdibujan los sujetos responsables, y en apariencia nadie tiene el control para actuar y a nadie le corresponde específicamente la responsabilidad para resolver y atender los problemas que se presentan dentro de la institución.

\section{La cadena de errores}

En la cadena de errores encontramos principalmente diagnósticos erróneos o tardíos y errores en las intervenciones quirúrgicas, en donde hay una suerte de superficialidad en la atención. Puede ocurrir, por ejemplo, que un médico desacredite la sintomatología del paciente, otro realice un mal diagnóstico, otro llene mal la historia clínica, el que le sigue no la lea y un último dé un tratamiento inadecuado o cometa errores en una intervención quirúrgica. Esta cadena de errores es propia de un ejercicio burocrático de la práctica médica que pondera la adaptación de esta a la lógica interna de la estructura del sistema de salud por encima de la atención al paciente. En esta cadena se encuentran involucrados diferentes médicos que individualmente cometen algún tipo de error y que, en conjunto, contribuyen al desenlace del suceso, en la mayoría de los casos con la muerte del paciente o con consecuencias graves, como la pérdida de órganos o de funcionalidad de estos y que generan incapacidades totales o parciales permanentes.

En los relatos encontramos formas de violencia institucional con discursos y prácticas de clasismo, discriminación y autoritarismo. Cabe destacar que muchas de las causas en la búsqueda de atención refieren a padecimientos comunes, por ejemplo, la diabetes o la hipertensión, dos de las principales enfermedades en México, es decir, no son enfermedades difíciles de diagnosticar. En ninguno de los casos se encontraron diagnósticos de patologías raras o de diagnósticos diferenciales complicados. Encontramos que los pacientes fueron atendidos hasta por 22 médicos, en los cuales la CNDH va señalando cada uno de los errores específicos de cada agente institucional y que en conjunto contribuyeron a las consecuencias fatales de los casos analizados.

\section{La violencia obstétrica}

La violencia obstétrica es una forma específica de violación a los derechos humanos y reproductivos de las mujeres, la cual se genera en los servicios de salud en la atención durante el embarazo, parto o puerperio. Son conductas que han 
permanecido invisibilizadas y que se relacionan con la negación de acceso a los servicios de salud (Grupo de Información en Reproducción Elegida, 2015). La tipificación de la violencia obstétrica ha coadyuvado en la visibilización de este tipo de violencia ejercida contra mujeres y, a su vez, evidencia el papel del Estado por acción u omisión en los servicios públicos. En las demandas de las mujeres ante la $\mathrm{CNDH}$, en materia de salud, se evidencian diversas formas de violencia con desenlaces graves como son la mortalidad materna y la perinatal, la esterilización y anticoncepción forzadas, así como parir fuera de la sala de parto.

\section{La exclusión}

El concepto de exclusión social se refiere a la privación de los derechos y libertades básicas que sufren amplios grupos poblacionales (Subirats, 2004). Los casos descritos en las gacetas configuran, en general, a un sujeto excluido y dominado. En este sentido, teorizamos respecto a la exclusión de los sujetos dominados en su condición de sumisión y su sentido de agencia mínimo o inexistente. (Auyero, 2013).

En el análisis de los grupos poblacionales veremos cómo se concatenan las cuatro categorías mencionadas.

\section{Perfiles poblacionales}

Como señalamos antes, hemos organizado el análisis que sigue clasificando las recomendaciones en cuatro tipos de población. Presentaremos nuestro análisis diferenciando tales grupos.

\section{Mujeres embarazadas}

Las mujeres embarazadas representan el grupo mayoritario de los perfiles poblacionales y constituyen el $67.4 \%$ del total de las mujeres. Un elemento a señalar es que en el $13.2 \%$ de los casos la investigación fue iniciada por oficio, es decir, que la afectada no fue quien interpuso la queja sino que apareció en los medios de comunicación y, a partir de este hecho, la CNDH inició la investigación. En muchos casos las mujeres no denuncian porque saben que no va a servir de nada en su proceso de aprendizaje y anulación. Confirmamos que los casos de las mujeres embarazadas operan a través de estructuras de opresión múltiples y simultáneas de clase, raza y género (Davis, 2005).

La gran mayoría de los casos (73.6 \%) culminó en la muerte de las mujeres embarazadas o de sus hijos o de ambos seres. El porcentaje más alto, $53.8 \%$, es la muerte de bebés; en 7.7 \% casos murieron la madre y su bebé, y en $11 \%$ las mujeres 
murieron dejando a sus hijos sin protección materna. Los tres escenarios son muy graves con diferentes implicaciones sociales.

\section{Muerte del bebé}

La política pública ha focalizado su vigilancia sobre la mortalidad materna y la importancia que tiene que una mujer muera durante el periodo del embarazo, parto o puerperio por causas evitables. Pero un indicador que ha tenido menos impacto es la mortalidad perinatal $l^{5}$ y la importancia que tiene que, aunque la mujer sobreviva, pierda al hijo o hija que esperaba. "Ellos al momento de entregarnos a nuestros hijos en una cajita ni siquiera alcanzan a comprender la magnitud del daño que nos causan" (Guadalupe, Zacatecas. Tribunal Simbólico-GIRE).

Identificamos cómo en el campo médico, se analiza la muerte perinatal en relación con los factores etiopatológicos con un enfoque desde la teoría de la causalidad y se explican las causas médicas por las cuales mueren los "productos" (Huiza, Pacora, \& Buzzio, 2003). Sin embargo, ¿por qué suceden dichas causas? Efectivamente, en las recomendaciones de la CNDH se presentan diversos diagnósticos clínicos citados en el análisis médico como sufrimiento fetal, asfixia neonatal, hemorragia transvaginal severa, etc. Pero cómo y por qué es que se generan dichas consecuencias, cuando no forman parte de la historia natural del embarazo, es lo que no nos relatan los análisis médicos de la causalidad. Dicho en forma breve: en las síntesis de los casos analizados, identificamos que dichas causas médicas efectivamente se presentaron, pero que muchas de ellas fueron el resultado de la falta de atención oportuna, es decir, que fue durante la espera donde se generaron dichas consecuencias, pues fueron casos de negligencia, de rechazo o retraso en la atención, de abandono y de descrédito de la sintomatología de la mujer y su consiguiente anulación.

En varios testimonios, las mujeres hacen referencia a la culpa, dado que los médicos suelen culparlas a ellas de las consecuencias, además de hacerlas sentir rechazadas, ignoradas y menospreciadas (Werner \& Malterud, 2003).

Por otro lado, en algunos casos encontramos quejas relacionadas con la desaparición del cadáver del bebé. El discurso médico utiliza la denominación del bebé como "producto" o como "tejido muerto", dependiendo de la etapa de desarrollo en la cual se encuentre al momento de su muerte. La Real Academia Española define producto como "cosa producida", en este sentido analizamos la propia concepción del "no-ser" que probablemente influye en el manejo poco cuidadoso del cuerpo.

5 La muerte perinatal se considera después de la semana 28 del embarazo hasta el séptimo día del posparto. 
De tal suerte, advertimos formas lingüísticas que se constituyen a partir de la ruptura con el mundo emocional, cosificando y objetivando al cuerpo del bebé como un cuerpo-objeto que no reconoce el cuerpo entendido como una construcción simbólica en torno a la identidad y personificación que le otorga la madre (López García de Madinabeitia, 2011). De tal forma, el discurso y accionar médico medicaliza los cuerpos anulando el apego emocional de la madre, la construcción social de la familia, la ritualidad en el proceso de la muerte y del duelo. Encontramos también una conexión entre el encubrimiento institucional y el velo místico en la desaparición de los cuerpos donde el personal sanitario y administrativo no da explicaciones, no saben qué pasó y se difumina la responsabilidad individual e institucional.

\section{Muerte de la madre y el bebé}

Respecto al descrédito de la sintomatología de la mujer, encontramos un patrón de violencia donde el dolor femenino es menospreciado y hay una anulación de los síntomas referidos por las mujeres. Las embarazadas dicen no sentir al bebé, tener sangrado o dolor, signos de infección, etc. y los médicos las mandan a caminar, las pelotean incluso cuando por factores como la edad, antecedentes de preeclampsia, infección, oligohidramnios (volumen deficiente de líquido amniótico asociado con complicaciones maternas y fetales) o cualquier otro indicador, tendrían que ser clasificadas y tratadas como pacientes de alto riesgo. Por ejemplo, en la recomendación 25/2013, una mujer de 15 años buscó cinco veces atención. El ginecólogo del nosocomio que valoró a la paciente omitió generar una historia clínica y tampoco informó sobre la salud del bebé. En el informe resaltó indicios de infección en vías urinarias, pero no valoró el riesgo para el bebé. En una segunda visita el mismo médico omitió el antecedente de infección. El ginecólogo de la clínica rural detectó la infección y la trató, pero no catalogó el embarazo como de alto riesgo y no la hospitalizó aun con la infección. Después, un médico pasante tampoco valoró el embarazo como de alto riesgo, lo cual complicó el estado de salud de la paciente al no ser hospitalizada. Un cuarto médico, ginecólogo, omitió suministrar antibióticos y ordenar ultrasonido. Ninguno de los médicos hizo un diagnosticó adecuado, en el cual debieron extraer al bebé para salvar la vida de la paciente. El bebé llevaba 10 días muerto en el vientre de la madre, lo que le provocó una infección y ella también murió.

En este caso se aprecian la interrelación de nuestras cuatro categorías de análisis: la exclusión y la cadena de errores donde se evidencia que la mujer acudió en cinco ocasiones y fue atendida por cuatro médicos, incluso por alguno con especialidad en ginecología, en las cuales fue mal diagnosticada y mal atendida; así también identificamos formas de violencia obstétrica que anulan a la mujer con acciones de indiferencia frente a su solicitud de atención, con un evidente descrédito de 
la sintomatología y un aplazamiento de su atención urgente, lo que la situó en un largo proceso de espera y de peloteo que agravó su condición hasta que finalmente murió junto con su bebé.

\section{Muerte de la madre}

La muerte de la madre refleja una mezcla de clasismo, inequidades, y discriminaciones que tienen implicaciones materiales para la familia, la cual, además del duelo, tiene que resolver el reacomodo en los roles, el cuidado, la atención y los gastos de manutención del bebé nacido. Todo ello, aunado a las condiciones de precariedad y exclusión, dificulta el escenario familiar.

En la recomendación 66/2010 una mujer embarazada acudió a consulta con el ginecólogo. Ahí manifestó que tenía un crecimiento en su seno, pero le dijeron que se trataba de una bolita de grasa y que su estado de salud sería "favorable". Un año después, regresó con el mismo ginecólogo, quien la revisó por estar embarazada otra vez, pero omitió el crecimiento del tumor en el seno. Un tercer ginecólogo omitió los indicios de cáncer, no leyó el expediente clínico y se enfocó en el embarazo de la paciente. Tras dar a luz, dos meses después regresó a consulta y un médico la remitió al tercer nivel de atención con urgencia ante los visibles síntomas de metástasis. Un mes y medio después murió y dejó a cuatro hijas: una recién nacida, una de un año, una de 6 y otra de 10. Los familiares indicaron que aunque los médicos ya tenían antecedentes del crecimiento de una glándula mamaria, le informaron a la víctima que habían "perdido" su expediente clínico.

En este caso nuevamente podemos distinguir la cadena de errores en las cuales la mujer acude a los servicios de salud y distintos médicos van omitiendo signos y síntomas, en los cuales se refleja la superficialidad de la atención y el descrédito de la sintomatología de la mujer.

Otro elemento a destacar son los problemas en relación con los expedientes clínicos. Encontramos mecanismos institucionales y del gremio médico relacionados con el encubrimiento de los errores, en los cuales las autoridades se niegan a colaborar con la investigación de la CNDH, no contestan la queja e incluso niegan los expedientes con la excusa de que se "extraviaron" o se "depuraron por error", algunos son expedientes incompletos e ilegibles en los cuales no se puede establecer ni a los responsables ni las acciones realizadas. Al igual que en la violencia obstétrica, la CNDH emitió la recomendación general 29/2017: "Sobre el expediente clínico como parte del derecho a la información en servicios de salud", al identificar que dichos sucesos no eran casos aislados sino que se repetían casuísticamente. 


\section{Parir fuera de la sala de parto}

En este tenor, otro elemento de análisis es el abandono de las mujeres, las cuales ya habiendo ingresado a los servicios de salud después de varias horas de espera y de mandarlas a caminar, a algunas, en repetidas ocasiones -peloteo-, acabaron pariendo en el baño, en la vía pública, en la sala de espera, en el patio o en algún otro lugar fuera de la sala de parto, pero en el interior del servicio o en la inmediaciones de este. Dicha situación la encontramos en casi el $10 \%$ de las mujeres embarazadas.

Por ejemplo, la recomendación 50/2014 correspondió a un caso iniciado de oficio en el cual la mujer acudió al nosocomio desde las seis de la mañana, los médicos la mandaron cuatro veces a caminar y acabó pariendo a las 11 de la noche en la banqueta afuera del servicio. Este caso se denunció a través de las noticias, ahí recogimos el testimonio del esposo de la agraviada:

La mera verdad, mi señora vino desde las 6 de la mañana al hospital. La checaron y le dijeron que todavía no le tocaba. Pasaron como dos horas y los dolores eran más fuertes y le decían que más al rato, y así pasaron tres horas y nada. Mejor nos fuimos para la casa...se puso bien mala, se le reventó la fuente, estuvo sangrando y me la lleve al hospital. La metí cargando y (los doctores) decían que todavía le faltaban cuatro centímetros y me hicieron que la sacara... serían entre las nueve o diez y media de la noche cuando mi esposa se hincó porque ya no aguantaba los dolores. (Matías, 2014)

\section{Esterilización y anticoncepción forzada}

Otro elemento que forma parte de la violencia institucional y violación del derecho a la salud son las formas de violencia que convergen en la esterilización y anticoncepción forzadas respecto a la violación de la autonomía en la decisión de las mujeres. Estas se entretejen por medio de amenazas, coacción y condicionamiento de para atenderlas. En la recomendación 61/2016, una mujer embarazada de 19 años indígena zapoteca de Oaxaca buscó atención. En la institución de salud omitieron realizarle varias pruebas, le hicieron un diagnóstico inadecuado, la abandonaron durante 13 horas, su bebé murió y después le hicieron una salpingoclasia de manera forzada. El esposo se enteró que a su mujer le habían hecho una salpingoclasia y que "el personal médico nunca les hizo referencia, ni les explicó los alcances y consecuencias de dicho procedimiento". En el relato se especifica que recibió un trato "grosero" y que incluso recibió "hasta regaños".

Después de la publicación en notas periodísticas, el esposo señaló que en una reunión se le "explicó que efectivamente hubo un error en la atención brindada a la agraviada, pero que era necesario que desistieran de cualquier acción o demanda legal, accediendo a firmar tal petición a fin de evitar cualquier inconveniente en 
la atención de la mujer". Este es un ejemplo recurrente en torno a las múltiples opresiones atravesadas por la clase, el género y la etnia, en donde las mujeres indígenas "sufren un trato inhumano, impersonal, agresivo, sin derecho a explicaciones" (Rovira, 1997, p. 221). Para agravar aún más la situación de violencia y de autoritarismo, a algunas mujeres las esterilizan de manera forzada, después de perder a su bebé, aunque a veces sean madres primerizas o muy jóvenes.

\section{Mujeres no embarazadas}

Las mujeres de este grupo se encuentran, en su mayoría, después de la edad reproductiva. Casi el $35 \%$ de las mujeres están en el grupo de 65 años y más, aquí se concentra el mayor número de personas adultas mayores de toda la muestra.

El desenlace, en la mayoría de los casos, es, al igual que en otros grupos, la muerte $(73 \%)$, pero en estas mujeres hay un mayor número de incapacidades permanentes que incluyen la muerte cerebral, la pérdida de órganos y las amputaciones de extremidades, entre otras. Es decir, las consecuencias y la afectación en la calidad de vida, para la agraviada y su familia, se evidencian en la cotidianidad y en la carga económica social y laboral, más aún si están en una etapa avanzada de la vida.

La investigación respecto a la violencia se ha focalizado en las mujeres embarazadas, incluso desde los reflectores de los medios de información, pero las mujeres que están fuera de la etapa reproductiva están invisibilizadas, a tal punto que no hay registro de ningún caso "por oficio". En ese tenor, relacionando la exclusión con el poder disciplinador y una agencia disminuida, encontramos en la recomendación 35/2010 el caso de una señora que interpuso una queja por dos eventos distintos. Es decir, la señora sufrió negligencia dos veces; la primera ocurrió en 2007, la segunda dos años después. Fue hasta que la violentaron por segunda ocasión que decidió quejarse. Esto quiere decir que la primera vez no fue registrada a su debido tiempo. No sabemos cuántos casos existirán en los que la víctima no se ha quejado por ningún medio, ni qué probabilidades existen de que una persona sufra dos veces negligencia y que no se queje por ninguna de las vías existentes.

El descrédito de la sintomatología no solo se presenta en la embarazada, en otros grupos también, incluso encontramos casos en los cuales el dolor es reasignado a una condición de "imaginario", a tal grado que se puede diagnosticar a una paciente con un padecimiento psiquiátrico antes que pensar que el dolor es real. En la recomendación 58/2011 la madre de la paciente de 16 años acudió al área de urgencias de la unidad médica, debido a un fuerte dolor abdominal de su hija. En dicho nosocomio, el médico adscrito la diagnosticó con colitis y le indicó de manera prepotente que comprara un medicamento para suministrárselo en la 
casa. El estado de salud de la paciente empeoró, y esta asistió al Hospital General donde el médico adscrito la diagnosticó con crisis de ansiedad y dio la orden de llevarla a atención psiquiátrica recetando diazepam, un ansiolítico y sedante que enmascaró el cuadro clínico con el cual cursaba la víctima. Sin mejoría alguna, la paciente y su madre regresaron a la unidad de medicina en repetidas ocasiones encontrándose con la atención prepotente por parte del mismo médico, quien ordena que la llevaran al Hospital Psiquiátrico. En dicho lugar informaron que no tenía relación el dolor abdominal con alguna enfermedad psiquiátrica, por lo que la canalizaron de urgencia al Hospital General, donde, a pesar de los intentos realizados no lograron salvarla y falleció debido a una pancreatitis aguda necro-hemorrágica e insuficiencia respiratoria. Dejó a una bebé de 5 meses. La señora refiere que el médico le dijo "... mira nada más como traes a tu hija...", “...ándale ya vete, llévate a tu hija a que la vea el psiquiátra o ¿qué vas a esperar a que se muera?", “ipor qué no la has llevado al psiquiátra?...”, “¿No has entendido que todo es mental, que no tiene nada?" (cursivas nuestras). En la última frase encontramos el descrédito de la sintomatología no solo como se da en la violencia obstétrica sino en general en los sujetos excluidos.

\section{Hombres}

Los hombres representan el $38.2 \%$ del total de los casos. Al contrario del perfil de las mujeres, casi el 3 \% está en el rango de edad de 1 a 14 años, es decir, la mayoría son niños o adolescentes, por lo cual podemos suponer que no fueron al servicio de manera autónoma. Esto concuerda con lo descrito en la bibliografía que ha identificado que los hombres utilizan menos los servicios de salud en comparación con las mujeres (Salgado-de Snyder \& Wong, 2007).

El 62 \% de los hombres murieron, pero además en este grupo es donde hay la mayor pérdida de órganos y amputaciones. También encontramos complicaciones de las enfermedades por la espera y la negación en la atención. Al igual que en el grupo de las mujeres no embarazadas, hay consecuencias de incapacidades parciales o totales permanentes.

Un elemento revelador es que en un $5.6 \%$ los órganos afectados fueron los testículos y en uno de ellos el pene. Sorprende la similitud en relación con los casos de esterilización forzada en las mujeres, donde al igual no existe el consentimiento por parte de los pacientes, no les explicaron o no les informan las consecuencias. Los médicos parecen no tomar en cuenta el desarrollo sexual, reproductivo y la construcción social de la masculinidad que afectará al agraviado con la extirpación quirúrgica de los órganos sexuales sin ponderar el mandato de masculinidad (Segato, 2018) que socialmente se les impone a los hombres. 
En los hombres, como parte de la exclusión encontramos grupos sociales con mayores condiciones de vulnerabilidad que propician formas de discriminación específicas, como son los casos de personas con VIH, migrantes y la población en centros de reclusión.

\section{Personas con VIH}

La mayoría de los casos de VIH están en este grupo, en ninguno de los grupos de mujeres hay casos relacionados con el VIH. A diferencia de los casos de discriminación en la población general, en la cual persisten aún algunos mitos en relación con las formas de contagio, al interior del campo médico situamos la percepción de amenaza persistente ante el riesgo de contagio, a pesar del alto grado de conocimiento de la enfermedad. En la recomendación 4/2002 se le negó la atención a un paciente en tres ocasiones por su enfermedad. En las recomendaciones 35/2009 y 42/2009, el equipo de hematología fue el responsable de la transmisión de VIH a niños de 10 y 13 años.

Después de dicho suceso, las víctimas, además, fueron violentados en su privacidad, exhibidos y discriminados por parte del personal médico y de enfermería, al punto de que pegaron una etiqueta de portador de VIH en su cama. De tal suerte, las personas con VIH son significadas e interpretadas a partir del estigma que les otorga un atributo desacreditador con una calidad diferente en oposición a la "normalidad" (Goffman, 2006) y, a diferencia de otros estigmas, el caso del VIH adquiere una significación de "monstruosidad" (Martínez \& Gavidia, 2006) que permea en los prejuicios y actitudes de los grupos profesionales de la salud coadyuvando en la negación de la atención (Reis et al., 2005). Encontramos formas de discriminación que no solo no son percibidas por el personal médico, sino que son justificas con el argumento de "darles un mejor trato", como fue en estos casos donde identificamos una etiquetación pública del estigma.

\section{Migrantes}

Para el caso de migrantes, destaca que en la base de datos solo encontramos dos casos. El primero, de un menor de edad y, el segundo fue recogido de oficio cuando el visitador estaba en la estación por otro motivo y al escuchar sobre el caso de la muerte de un migrante decidió indagar sobre esta. Podemos suponer que la ausencia en los casos de migrantes no es porque sean un grupo al que no se les violan sus derechos. Por el contrario, la violación flagrante de los derechos se presenta contra personas más expuestas a la violencia sistémica, con mayores obstáculos en el libre ejercicio de sus derechos, lo cual se suma a las dificultades que se presentan para iniciar procesos de demandas y de acceso a la justicia. 


\section{Personas en situación de reclusión}

Este grupo, por su condición de privación de libertad, tiene cuantiosas dificultades para poder ejercer sus derechos. De las 31 recomendaciones generales que ha emitido la CNDH, encontramos ocho en las cuales la autoridad a la que están dirigidas pertenecen al área de la salud y tres de esas ocho incluyen a las personas en situación de reclusión: las recomendaciones $12 / 2017$ y $18 / 2010$, y la $3 / 2002$ específicamente para las mujeres. En los casos identificamos que las problemáticas de violación del derecho a la salud, la negación de la atención y la caracterización de los errores de negligencia, imprudencia e impericia se intensifican. Los relatos dan cuenta de la asistencia al médico después de un tiempo prolongado, narran la búsqueda de atención varias veces sin que los asistan adecuadamente, sin que les hagan estudios durante un largo periodo de tiempo y donde generalmente se les atiende de manera paliativa. La espera es mucho más larga de días, meses, e incluso se encontró un caso de diez años , en la cual los pacientes son diagnosticados o tratados erróneamente y, cuando finalmente los atienden, las consecuencias son muy graves.

Al igual que en las mujeres, encontramos el descrédito de la sintomatología y su reasignación a la condición de "imaginario". Esto se acentúa aún más en la cárcel. A dos de las personas recluidas las diagnosticaron con trastornos mentales cuando eran cuadros de padecimientos comunes, incluso con un diagnóstico inexistente como el del médico de la recomendación 47/2008 que dijo que el paciente tenía "psicosis carcelaria".

Continuando con el análisis de la exclusión y dominación de los pacientes, analizamos también un ejemplo del velo místico que se presenta en relación con el desabasto de medicamentos y sus consecuencias, muchas de ellas fatales. En la recomendación 64/2012, el paciente acudió a la farmacia del hospital a solicitar el medicamento sugerido por su médico; sin embargo, después un largo tiempo le confirmaron que no tenían abasto de medicamentos, por tal razón la víctima acudió con el director de dicho nosocomio; mientras discutían el tema, la víctima sufrió un infarto al miocardio y falleció.

\section{Casos colectivos}

La recomendación 4/2004 corresponde un caso en el cual murieron 35 bebés atendidos en el mismo hospital de Comitán, Chiapas, una de las regiones más pobres del país. Fue un caso identificado de oficio. El segundo caso es la recomendación 25/2005, de 360 casos de VIH publicados en una lista en los espacios del nosocomio 
de forma que se expusieron los datos personales de los pacientes durante cuatro días; vulnerando, así, su derecho a la privacidad; similar a la recomendación en el grupo de los hombres citada anteriormente. El tercer caso es la recomendación 41/2007, sobre desabasto de medicamentos para el VIH, de igual manera ligada al análisis del velo místico. El cuarto caso es la recomendación 57/2011 en la cual un enfermero de manera independiente decidió poner un puesto de vacunación y el resultado fueron cuatro víctimas de las cuales murieron dos y uno quedó con daños irreversibles. Y el quinto caso es la recomendación 13/2016 que se emite por las deficientes condiciones en la situación de reclusión.

\section{Infraestructura ligada a burocracia}

A pesar de sostener que la violencia institucional y violación del derecho a la salud no se deben exclusivamente a problemas de infraestructura, también encontramos casos específicos de este tipo que, ligados a la incertidumbre y arbitrariedad de los procesos burocráticos, coadyuvan en las consecuencias graves por la falta de atención oportuna. Encontramos varios elementos de burocracia ligados a la arbitrariedad que terminaron en casos de negligencia, como la recomendación 38/2016 donde el director no firmó el acta de traslado urgente, porque se encontraba en una reunión y el desenlace fue la muerte del bebé; en la recomendación 58/2016 tampoco pudieron trasladar a una mujer embarazada, porque la ambulancia no tenía gasolina y no encontraban al chofer, sus familiares la trasladaron y la historia culminó con la muerte del bebé. En la recomendación 13/2014 un médico canalizó a un paciente a otro hospital y no lo aceptaron porque no tenían conocimiento de la referencia y porque no había camas; lo mandaron de regreso y, cuando volvió al hospital de origen, lo regresaron nuevamente. Murió en uno de los tres trayectos. Así, encontramos múltiples elementos relacionados con el cambio de turno, con reuniones, vacaciones o alimentación de los grupos de profesionales, firmas de documentos, etc.

Responsabilidad institucional vs responsabilidad individualizada del menor eslabón jerárquico

De igual modo, es preciso establecer la responsabilidad institucional, ya que en múltiples ocasiones se culpa a los médicos pasantes o a los residentes, sin establecer que la responsabilidad tanto de los pacientes, como la supervisión de las acciones de los subordinados es de los médicos adscritos. Se sabe que en la jerarquía médica se reproducen formas de violencia como parte del currículo oculto del campo médico. Por lo tanto, dicha problemática forma parte de un contexto más complejo que incluye la insuficiencia de recursos y de la infraestructura necesaria para la atención, así como las condiciones laborales precarias en las cuales trabaja gran 
parte del personal de salud en el sector público. En ese sentido, dichas condiciones permean el funcionamiento y la atención en el sistema de salud.

\section{Conclusiones}

Esta investigación buscó generar evidencias sobre elementos que puedan constituir obstáculos en el ejercicio del derecho a la salud. A la vez, su propósito fue documentar las violaciones de dicho derecho y caracterizar las principales formas de violencia institucional que sufren el público usuario de los servicios públicos de salud en la búsqueda de atención médica. Hemos tratado de identificar los patrones de violencia en la violación del derecho a la salud y las conductas invisibilizadas de exclusión de carácter estructural en las recomendaciones emitidas por la $\mathrm{CNDH}$ del 2000 al 2016 en esta materia.

Es necesario resaltar que los datos analizados tienen un sesgo respecto a la gravedad de los casos, ya que son casos tipificados como violaciones del derecho a la salud y no se toman en cuenta los miles de casos "exitosos" -que debe haberlos-; pues la importancia del análisis radica en identificar y poder transitar hacia modelos de salud que tomen en cuenta contextos sociales específicos y que no respondan únicamente a lógicas burocratizadas y burocratizantes reproductoras de la dominación y del sometimiento, y que ponen en peligro la vida de sus pacientes.

Debatimos contra los argumentos que sustentan que la mayoría de los errores, la mala praxis, es decir, la falta de apego a la lex artis se debe, principalmente, a los múltiples problemas de infraestructura de los servicios públicos de salud, los cuales incluyen la falta de personal, las carencias de insumos y, por supuesto, servicios rebasados respecto a la demanda (Santamaría-Benhumea et al., 2014). A pesar de dichos elementos, que efectivamente influyen en la atención, un hallazgo de la investigación es que en un gran número de casos de las quejas presentadas ante la CNDH el argumento de la infraestructura no es el elemento principal de los acontecimientos narrados, sino aquellos elementos relacionados con el clasismo, el racismo y el sexismo, perpetrados a través prácticas de discriminación, de autoritarismo y de exclusión social. Dichos elementos los identificamos a través del análisis de la espera, la cadena de errores, la violencia obstétrica y la exclusión.

En este sentido, en la espera identificamos el tiempo como un factor primordial. En el tiempo se entretejen la espera y la exclusión, determinantes en las consecuencias de salud. En gran parte de los casos de la CNDH, de haber recibido un diagnóstico o una atención en el debido tiempo, podrían seguir con vida o sin complicaciones físicas graves. En la espera se reproducen formas de dominación donde cada paciente aprende a esperar de forma sumisa y "cumplir pacientemente 
con los requisitos arbitrarios, ambiguos y siempre cambiantes que les impone el Estado" (Auyero, 2013, p. 24).

En la cadena de errores identificamos que se constituyen principalmente por la negación del servicio, la omisión de información a pacientes, problemas con el expediente clínico, los diagnósticos incorrectos, el abandono y los errores en las intervenciones quirúrgicas que, como pudimos constatar en los casos que revisamos, la mayoría de las veces terminan en la muerte o con consecuencias graves para pacientes y familiares.

En relación con la violencia obstétrica, en las mujeres embarazadas, grupo mayoritario, destacamos que la OMS ha sustentado que muchos de los casos de mortalidad materna y perinatal se deben a que estas no reciben atención médica antes, durante y después del parto; hace hincapié en que no llegan a los servicios, pero no evidencia los casos en que las mujeres sí llegan y no reciben atención o la reciben de forma negligente (OMS, 2018). Uno de los argumentos esgrimidos por el gremio médico que culpabiliza a la mujer y coadyuva, de paso, a descalificar la medicina tradicional y a las parteras, son las complicaciones que sufren las mujeres por no buscar atención en los espacios de la medicina hegemónica. La mortalidad materna se ha explicado a partir del modelo de las tres demoras: 1) retraso en la búsqueda de ayuda, 2) demora en la llegada y 3 ) tardanza en la respuesta por parte de los servicios de salud (Loza, 2013). A partir de la revisión de las recomendaciones, en el presente análisis ubicamos a la gran mayoría de los casos en la tercera demora, dado que todas las mujeres llegaron a los servicios de salud y dentro del mismo más bien hubo una negación de la atención, un retraso en la atención oportuna o el abandono de la mujer a pesar de sus "súplicas" para ser atendida. Esta situación se encuentra en la caracterización general de los relatos en donde la espera dentro de los servicios durante horas, e incluso días, culmina en la muerte.

En los casos analizados, las mujeres, al llegar a los servicios, se encuentran con profesionales de la salud que les exigen "presentar más sangrado y mayores dolores" (recomendación 52/2015). En este sentido, encontramos que como parte de la violencia obstétrica hay una normalización del riesgo y un aplazamiento de la atención médica urgente que se representa en la indiferencia ante las solicitudes de las mujeres y su anulación. La percepción del dolor en las mujeres, al igual que otros síntomas, está medicalizada, es decir, se contempla dentro del paradigma dominante científico en el cual los médicos hacen una construcción naturalizada del dolor en el proceso del parto y en donde las mujeres emprenden una lucha por la credibilidad en un debate entre la dominación y la agencia. En este debate ellas tienen que convencer a sus médicos. Se normalizan las urgencias y hay un descrédito de la sintomatología, a pesar de signos de alarma desde los propios parámetros alópatas. Así, 
los profesionales de la salud les exigen tener más dolor, es decir, exigen el aumento en la magnitud de un síntoma subjetivo que no puede medirse, ni conocerse con certidumbre y exactitud; pero que en general determinan que no es "suficiente", como se ha descrito en otras investigaciones donde el dolor en la mujer es "subvalorizado, subdiagnosticado y subtratado" (Bernardes, 2014).

Como parte del análisis de la exclusión, pudimos constatar en los relatos que en la violación en el ejercicio de los derechos se reproducen diversas formas de violencia institucional, en las cuales el sistema de salud ata y excluye a sus usuarios e incluso, en algunas ocasiones, los extingue (Bialakowsky, 2006).

Es primordial aclarar que no nos proponemos la criminalización del acto médico, porque tenemos claro que, efectivamente, existen graves problemas de infraestructura y carencias de recursos en el sistema de salud público. Estas limitaciones dificultan la adecuada y oportuna atención médica. Sin embargo, sostenemos que es necesario evidenciar la falta de protección del público usuario frente a la violación del derecho a la salud, así como la violencia institucional que se ejerce durante el proceso de atención. Es necesario identificar el papel del Estado en las instituciones de salud pública, ya que "los llamados a la 'humanización' de los médicos de forma individualizada desvinculan la responsabilidad institucional que subyace en las formas de violencia ejercida sobre los pacientes" (Rueda E, 2013 p. 94). Además, es importante señalar los mecanismos institucionales que obstaculizan el ejercicio de los derechos, lo que, a su vez, dificulta la reparación del daño y la adecuada indemnización de pacientes. Podríamos apuntar que el sistema se preocupa más por asegurar su permanencia y su funcionamiento que por la atención que brinda.

Una limitante fue trabajar con fuentes secundarias, lo que no nos permitió indagar más allá de lo que está plasmado en los relatos de las recomendaciones, salvo en aquellos casos donde encontramos los testimonios en los medios de comunicación. Este trabajo fue un primer acercamiento de análisis de la temática planteada. Sin duda, es necesario investigar, en un segundo momento, a partir de nuevas líneas de indagación en torno a la espera como elemento de dominación de pacientes, la caracterización de formas de violencia obstétrica, la exclusión y la cadena de errores desde una aproximación etnográfica que permita profundizar en torno al descrédito de la sintomatología y su relación con padecimientos psiquiátricos tanto en hombres como en mujeres, el trato de los cuerpos de los bebés muertos y otros elementos identificados a lo largo del análisis planteado por medio, por ejemplo, de entrevistas a profundidad. 


\section{Agradecimientos}

Esta investigación se realizó gracias al Programa de Becas Posdoctorales en la UNAM. Agradezco en especial la valiosa colaboración de Carlos Ricardo López Gómez en la redacción final del texto, a Marco Antonio Ávila Peña por el apoyo en la base de datos y a las reflexiones de ambos, las cuales enriquecieron sustancialmente este trabajo.

\section{Referencias}

Armida, M. J., Cassino, M., Ciarniello, L., \& Witis, R. (s. f). Los derechos humanos frente a la violencia institucional. Recuperado de http://www.bnm.me.gov.ar/giga1/documentos/EL005061.pdf

Auyero, J. (2013). Pacientes del Estado. Argentina: Eudeba.

Bernardes, S. (2014). Existirão enviesamentos de sexo nos julgamentos de dor de enfermeiro/as? Sim... mas nem sempre. Dor, 1, 34-40.

Bodelón, E. (2014). Violencia institucional y violencia de género. Anales de la Cátedra Francisco Suárez, 48, 131-155.

Comisión Nacional de los Derechos Humanos. (2017). Informe de actividades del 1 de enero al 31 de diciembre de 2017. México: Autor.

Davis, A. (2005). Mujeres, raza y clase. Madrid: Akal.

De Sousa Santos, B. (2014). Si Dios fuese un activista de los derechos humanos. Madrid: Editorial Trotta.

Goffman, E. (2006). Estigma. La identidad deteriorada. Buenos Aires-Madrid: Amorrortu editores.

Grupo de Información en Reproducción Elegida. (2015). Niñas y mujeres sin justicia . Derechos reproductivos en México. México: Autor.

Huiza, L., Pacora, P., \& Buzzio, Y. (2003). La muerte fetal y la muerte neonatal, 13-20.

López García de Madinabeitia, A. P. (2011). Duelo perinatal: Un secreto dentro de un misterio. Revista de la Asociación Española de Neuropsiquiatría, 31(109), 53-70. https://doi.org/10.4321/ S0211-57352011000100005

Loza, C. B. (2013). ¿La disminución de la mortalidad materna es un simple problema técnico de adecuación cultural? História, Ciências, Saúde, 20(3), 1082-1086. 
Martínez, E. N. R., \& Gavidia, N. G. (2006). Enfermedad y significación: Estigma y monstruosidad del VIH/SIDA. Revista de Ciencias Humanas y Sociales, 50(50), 9-28. Recuperado de http:// dialnet.unirioja.es/descarga/articulo/2476971.pdf\%5Cnfile:///Users/Marcos/Library/ Application Support/Papers2/Articles/2006/Martínez/2006 Martínez.pdf\%5Cnpapers2:// publication/uuid/03FB6C54-0227-4069-8E49-6AB38DE1BAD3

Matías, P. (2014). Mujer da a luz en banqueta por negligencia médica...otra vez en Oaxaca. Proceso.

Perelman, M., \& Trufó, M. (2017). Violencia institucional. Tensiones actuales de una categoría política central. Argentina.

Reis, C., Heisler, M., Amowitz, L. L., Moreland, R. S., Mafeni, J. O., Anyamele, C., \& Iacopino, V. (2005). Discriminatory attitudes and practices by health workers toward patients with HIV/AIDS in Nigeria. PLoS Medicine, 2(8), 0743-0752. https://doi.org/10.1371/journal. pmed.0020246

Rovira, G. (1997). Mujeres de maíz. México: Ediciones Era.

Rueda, E. A. (2013). Salud, violencia estructural y ley estatutaria: Un vistazo rápido a nueve patologías estructurales. Anamnésis Revista de Bioética, 8, 91-95.

Salgado-de Snyder, V. N., \& Wong, R. (2007). Género y pobreza: Determinantes de la salud en la vejez. Salud Pública de México, 49(1), 515-521. https://doi.org/10.1590/ S0036-36342007001000011

Santamaría-Benhumea, N. H., Garduño Alanís, A., de la Rosa Parra, J. A., Santamaría-Benhumea, M., Rueda Villalpando, J. P., \& Herrera Villalobos, J. E. (2014). Legal aspects of medical error in Mexico. Revista CONAMED, 19(1), 32-36. Recuperado de http://dialnet.unirioja.es/ servlet/articulo? codigo $=4730769 \&$ info $=$ resumen\&idioma $=$ SPA

Werner, A., \& Malterud, K. (2003). It is hard work behaving as a credible patient: encounters between women with chronic pain and their doctors. Social science $\mathbb{E}$ medicine (1982), 57(8), 1409-1419. Recuperado de http://www.ncbi.nlm.nih.gov/pubmed/12927471 